\title{
A GSM-Based Remote Temperature and Humidity Monitoring System for Granary
}

\author{
Xiao Xi Zheng ${ }^{1}$, Lian Rong $\mathrm{Li}^{1}$ and Shuai Fei Shao ${ }^{2}$ \\ ${ }^{1}$ Zhengzhou Technical College, Zhengzhou, Henan, China \\ ${ }^{2}$ Sippr engineering group CO.LTD, Zhengzhou, Henan, China
}

\begin{abstract}
A remote temperature and humidity monitoring system is designed based on the GSM technology and MSP430. With the digital sensor DSB1820 and SHT11, the temperature and humidity of the granary are detected, and these parameters can be adjusted with the controlling system to adapt various working conditions. Through the GSM system, the detected data could be sent to various monitoring devices, such as cellphones and laptops. These data can be used for data display, inquiry, controlling and storage at the remote terminals. The experimental results show that the system is convenient and concise, which meets the remote monitoring demand for the modern granary.
\end{abstract}

\section{Introduction}

Safe storage of grain is a big problem of people's livelihood, in the process of grain storage, easy to affected by storage environment temperature and humidity conditions, easy to fever, mildew and insect pests, etc.while improper granary of temperature or humidity control, , thus resulting in loss of food, to ensure the storage of food in good condition, must make the granary of the temperature and humidity control in a certain range.In order to improve the reliability of the system, GSM mobile communication network of wireless data transmission technology is applied to the granary monitoring system, which has less investment, quick opening, simple operation, low communication cost, and the advantages of reliability and coverage guaranteed, very suitable for small granary monitoring system of data transmission.

Based on a small grain granary as the research object, with high reliability, perfect function, strong antijamming capability, higher cost performance advantage of MSP430 MCU as the core controller, using the granary of temperature and humidity and the temperature and humidity of the grain in pile testing and the value passed to the single chip microcomputer, and then compared with the temperature and humidity of the preset threshold to decide whether to start the alarm circuit, by controlling the motor start-stop control of the temperature and humidity. Granary to make use of PC monitor and store data, the system uses the free GSM network as a way of information transmission, users can check to the food temperature and humidity data conveniently on the computer or mobile phone, realize the real-time remote monitoring of the temperature and humidity of a small Granary.

\section{The overall structure and function of the control system}

The granary intelligent monitoring system consists of the microcontroller core controller, real-time detection of temperature and humidity, GSM transmission of information of temperature and failure. MSP430 microcontroller as control core, through the external keyboard can easily set the telephone number and the monitoring center temperature and humidity, using temperature and humidity sensors to detect the monitoring point temperature and humidity inside the granary, using a temperature sensor to collect grain temperature, and the temperature and humidity data sent to the single-chip microcomputer software system for data processing, single chip digital output of the D/A conversion into A suitable analog to drive actuators to adjust temperature and humidity, so as to ensure the temperature and humidity of granary and grain are within the scope of the set.

The real time Temperature and humidity information is displayed through the LCD, Using GSM technology to transfer information to PC or mobile phone, it can be convenient to monitor information of granaries by $\mathrm{PC}$ or mobile phone. At the same time, fan open and alarm prompt system with related by administrators in the PC software and mobile phone can be convenient to monitor granaries. At the same time, the system with a fan and alarm prompt function, when the temperature exceeds the limit of the set automatically adjust temperature and

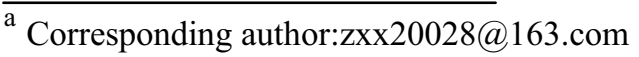


humidity, and sound and light alarm. Control system overall structure block diagram as shown in figure 1.

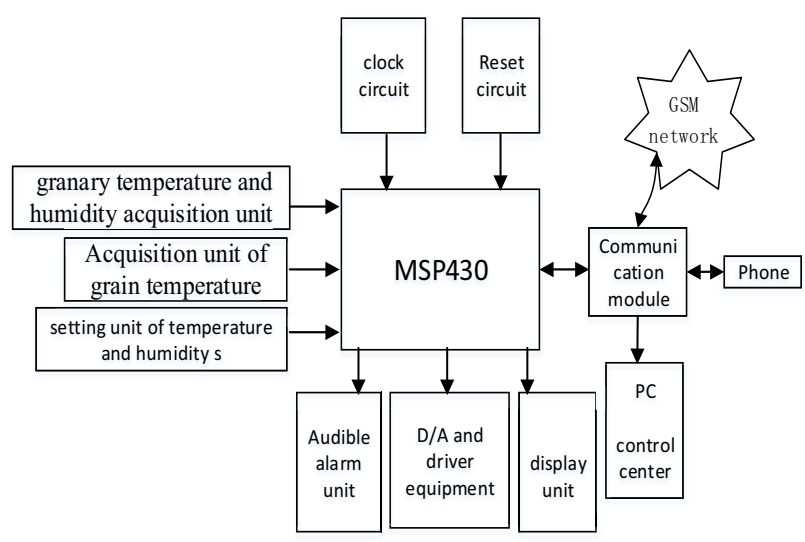

Figure 1. Control system overall structure block

\section{Hardware selection and design}

\subsection{Core controller}

MSP430 MCU by TI company launched a series of ultra-low power consumption microprocessor, it has the characteristics of ultra-low power consumption, there are five modes to choose from, low power consumption and wake up of time is very short, only need $6 \mathrm{mu} \mathrm{s}$, at the same time also has a strong processing capacity, rich onchip peripheral modules, flexible clock system, stable working condition, convenient and efficient way of development, etc. The above features make it become the preferred microprocessor. The system selects the MSP430F449 as the core controller, its minimum system diagram as shown in figure 2 .

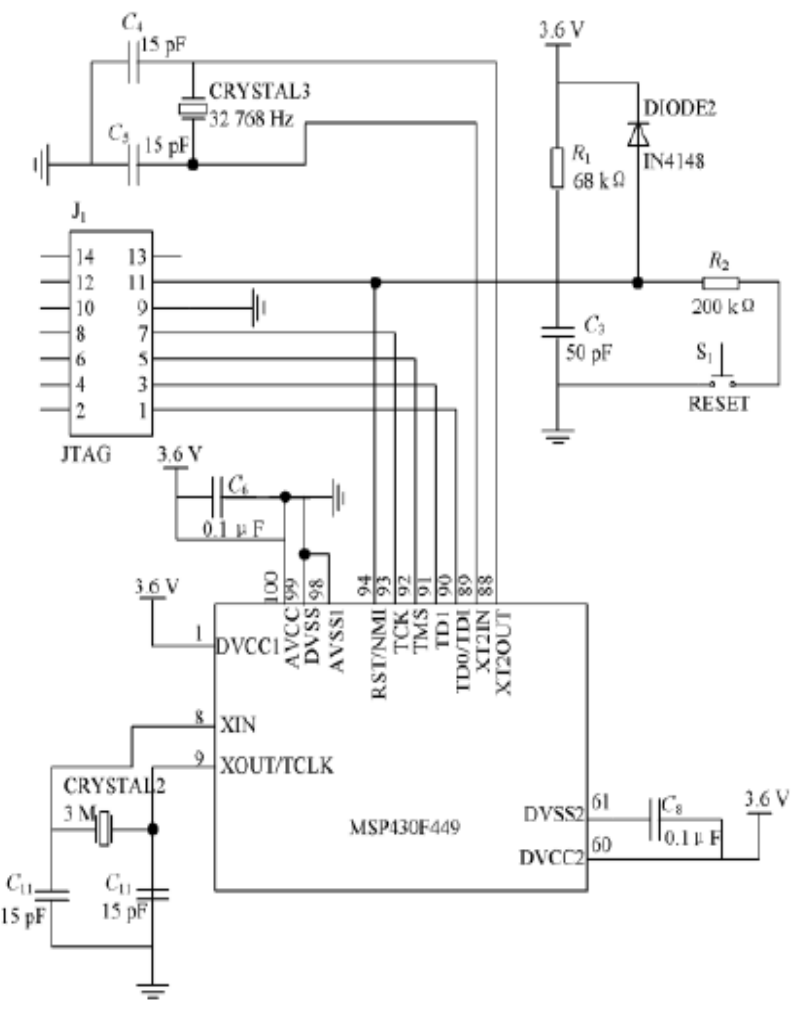

Figure 2. Minimum system diagram of MSP430

\subsection{Temperature and humidity acquisition unit of Granary}

Granary data acquisition module is responsible for the internal temperature and humidity information collection and data conversion, the system uses the temperature and humidity sensors SHT11 which was launched by sensirion company, the chip including temperature and humidity sensor, signal amplification processing, A / D conversion and I2C bus interface. Sensor SHT11 temperature range for $-40 \sim 123.8{ }^{\circ} \mathrm{C}$, for the resolution of the 14 is $0.01{ }^{\circ} \mathrm{C}$, humidity measurement range is $0 \sim$ $100 \%$ RH. Supply voltage of $2.4-5.5 \mathrm{~V}$, using two wire serial interface. Temperature and humidity data, temperature and humidity sensitive element respectively will converted to electrical signals, after the signal amplification, using A/D converter into digital signal, Then, through the bus interface and output to MSP430, MSP430 process and send signal. In order to avoid the signal conflict, one pull-up resistors in DATA access, at the same time one decoupling capacitor in the VCC \& gnd access. SHT11 and MSP430 interface circuit as shown in Figure 3.

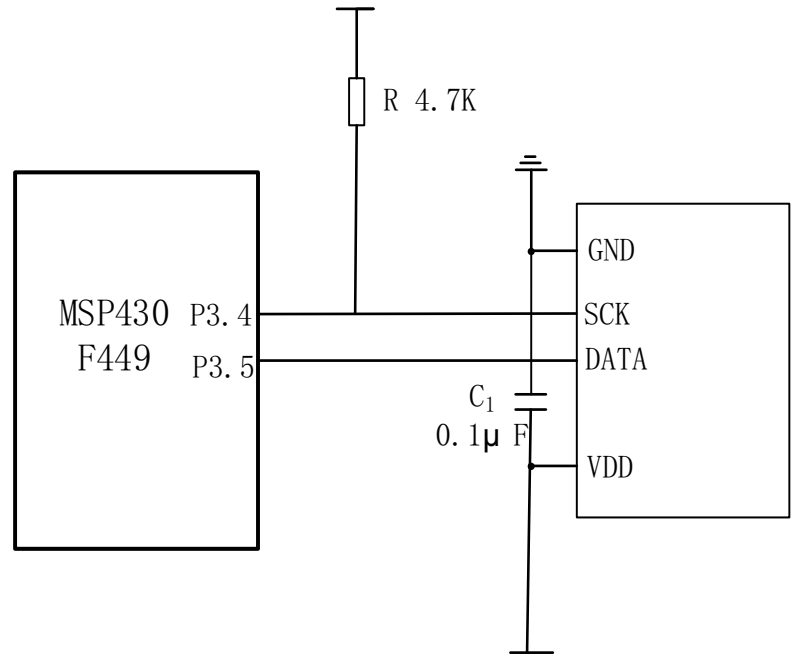

Figure 3. SHT11 and MSP430 interface circuit

\subsection{Acquisition unit of temperature in Grain bulk}

Using intelligent digital temperature sensor DS18B20 of Dallas to detecting the temperature of grain, can easily achieve multi-point temperature measurement, temperature measurement circuit as shown in Figure 4. DS18B20 temperature detection range of $-55^{\circ} \mathrm{C} \sim+125^{\circ} \mathrm{C}$, the maximum resolution of 12 , the detection accuracy of $\pm 0.5^{\circ} \mathrm{C}$. DS18B20 read and write information requires only one bus (ie, single-bus interface). the required energy of Temperature conversion is provided by bus, no external power supply. the temperature of the collected signal is converted to A digital output by DS18B20, directly connected to the microcontroller, don't need A/D conversion module, , which greatly simplifies the design of the circuit. Meanwhile, DS18B20 itself with the command set and memory, microcontroller by issuing 
control commands to read and write memory DS18B20 complete temperature measurement.

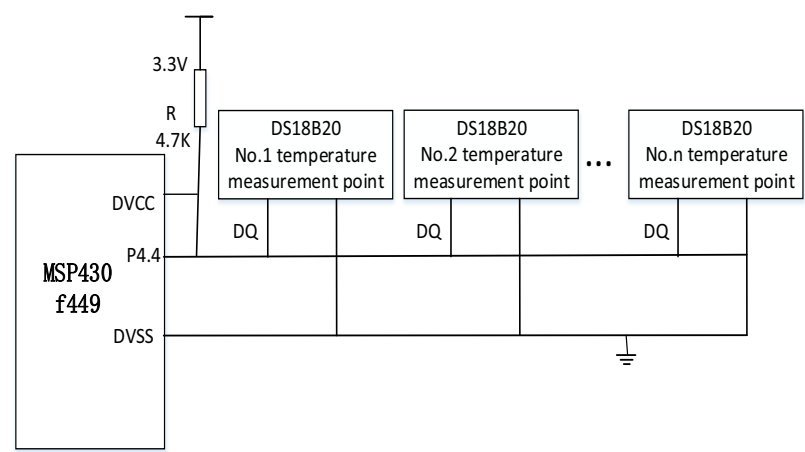

Figure 4. Temperature measurement circuit of Temperature sensor

\subsection{Design of GSM wireless communication module}

This design adopts common GSM wireless communication network as a data transmission channel, its coverage, transmission distance is not restricted. This design uses wireless transmission module of TC35i, which is composed of power supply module (ASIC), ZIF connectors, flash memory, antenna interface, etc. It's internal composition as shown in figure 5 .

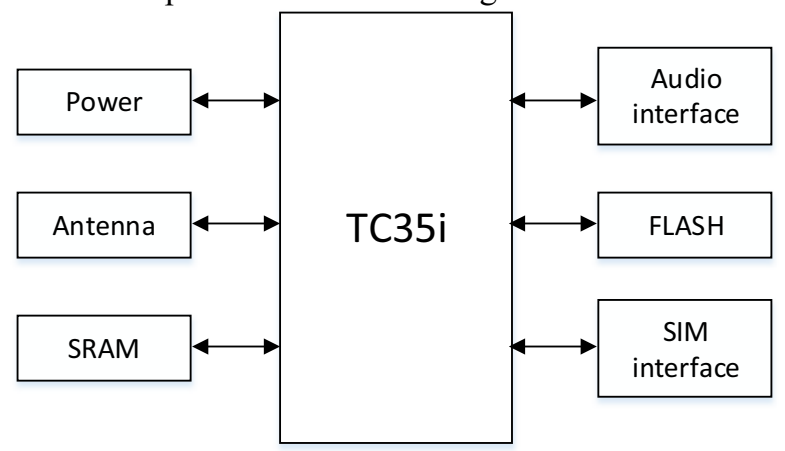

Figure 5. Internal component of TC35i module

Siemens TC35i module is to support Chinese short message and Work in EGSM900 and GSM1800 dual band, can carry both voice and data signals, this system is mainly to transmit data information. TC35i module connect SIM card reader and antenna respectively through the interface connector and antenna. TC35i data interface (CMOS level) can be two-way transfer instructions and data by the AT command, which supports Text and PDU format of the SMS (Short Message Service, Short Message), can be achieved on or off to restart and recovery by signal AT commands. TC35i for dc power supply range from $3.3 \mathrm{~V}$ to $4.8 \mathrm{~V}$, power supply circuit diagram as shown in figure 6 . Design, in order to reduce interference in the input point connected to filter capacitance; In order to reduce the output voltage stability, on the output side connected to three capacitance.

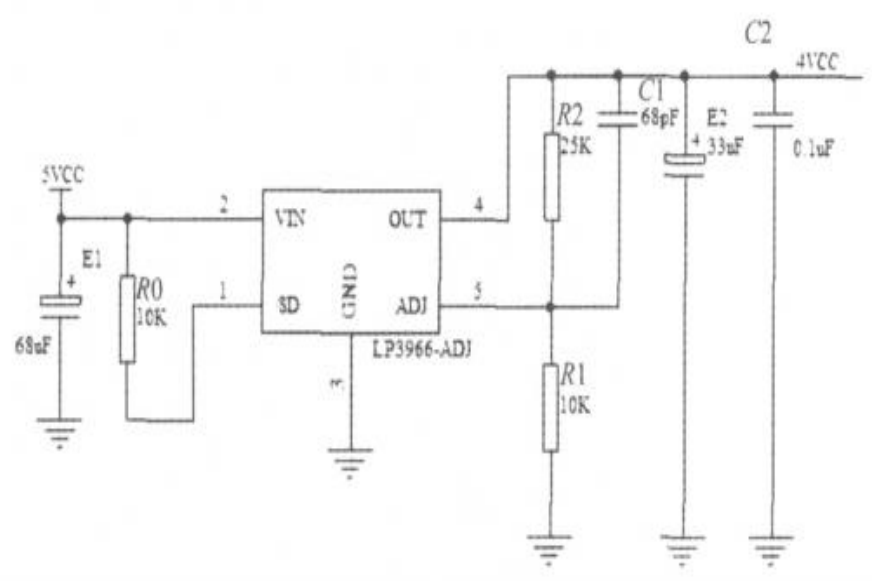

Figure 6. Power supply circuit diagram

TC35i has 40 pins, through a ZIF (zero resistance) socket connector leads. The 40 pin can be divided into power supply, input/output data, SIM card, audio interface and control 5 classes. Among them, $1 \sim 5$ for positive power input pins, voltage of 3.3 V. $6 \sim 10$ pin for power line ground;11, 12 pin for charging pins, to external rechargeable battery; 13 pin for output voltage to external circuit; 14 pins for BATTTEMP which connect the negative temperature coefficient thermistor, 15 pin is to start foot IGT, which connecting MCU P3.0 pin, through P3.0 making low pulse, to start the TC35i module; $16 \sim 23$ pin for data input/output, conforms to the ITU - TRS232 interface standards; 24 to 29 pins connect with SIM card; 30 32 for control pin; $33 \sim 40$ pin for the voice interface, used to connect the speaker, mike, headphones, etc. Unused pins of TC35i should be hung up as output, the input should be on, such as 21, 22 pin; SYNC instructions working condition of TC35i; power supply is connected, grounding and grounding; IGT pull up resistor connected to the microcontroller, can control the work state by MSP430. TC35i module interface circuit as shown in figure 7. The work level of serial port pin of TC35i is CMOS level, while the single chip computer is TTL level, using 74F07 to convert level to connect the TC35i serial port, only when the connection even good serial transceiver and TC35i diode lamp control side. It only need to do serial transceiver and TC35i diode lamp control when connecting both. 


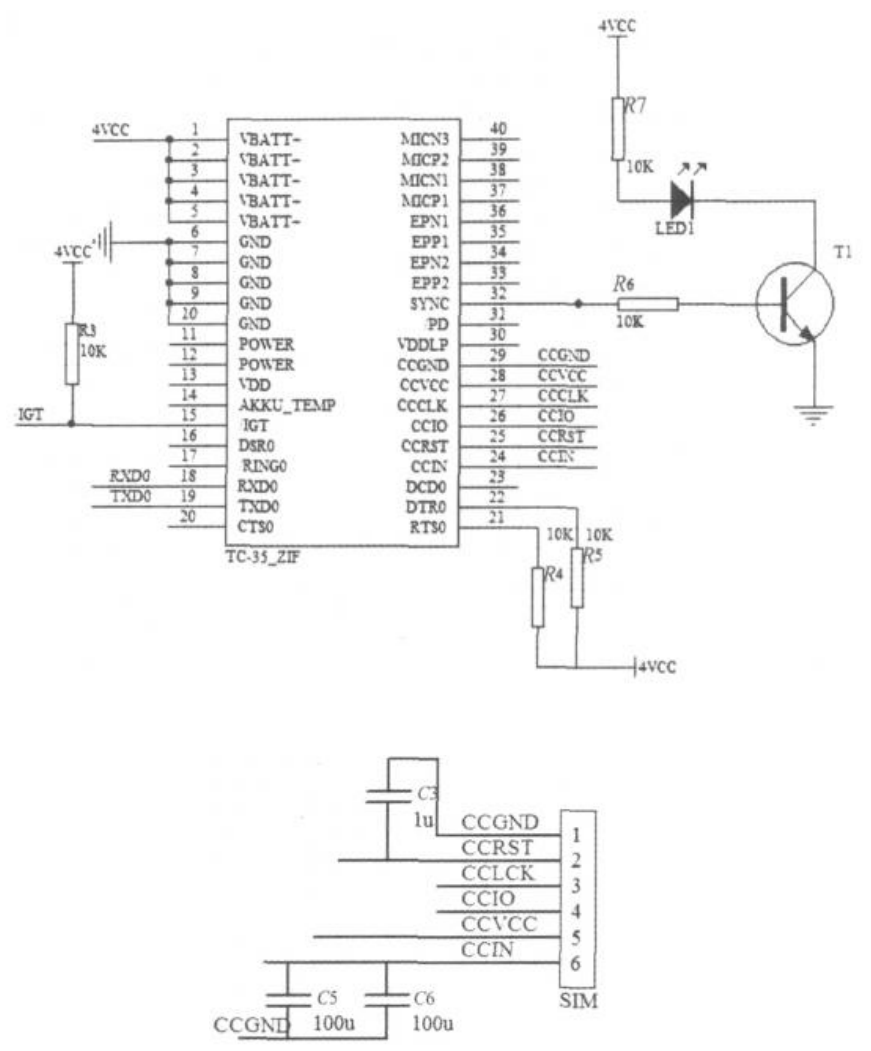

Figure 7. TC35i module interface circuit

\section{Software design of the system}

\subsection{Main program design of granary monitoring}

The function of main program for granary monitoring system are data analysis and processing. The design of system software is mainly composed of Initialization, temperature and humidity data acquisition and signal display device driver, signal processing, keyboard processing, data communication, etc. Program flow chart as shown in figure 8 . The initialization completed the functional configuration of the initial state of single-chip, open watchdog; Real-time data acquisition of temperature, humidity, alternating with single-chip computer transmission data, after data communication unit to complete communication. between the control system of temperature - humidity measurement and PC machine (or phone). When the measurement data detected by Singlechip is greater than the set threshold, single-chip send control signal in time, driving circuit control fan equipment such as opening and closing, when the temperature and humidity is not in the normal range, start the audible and visual alarm at the same time.

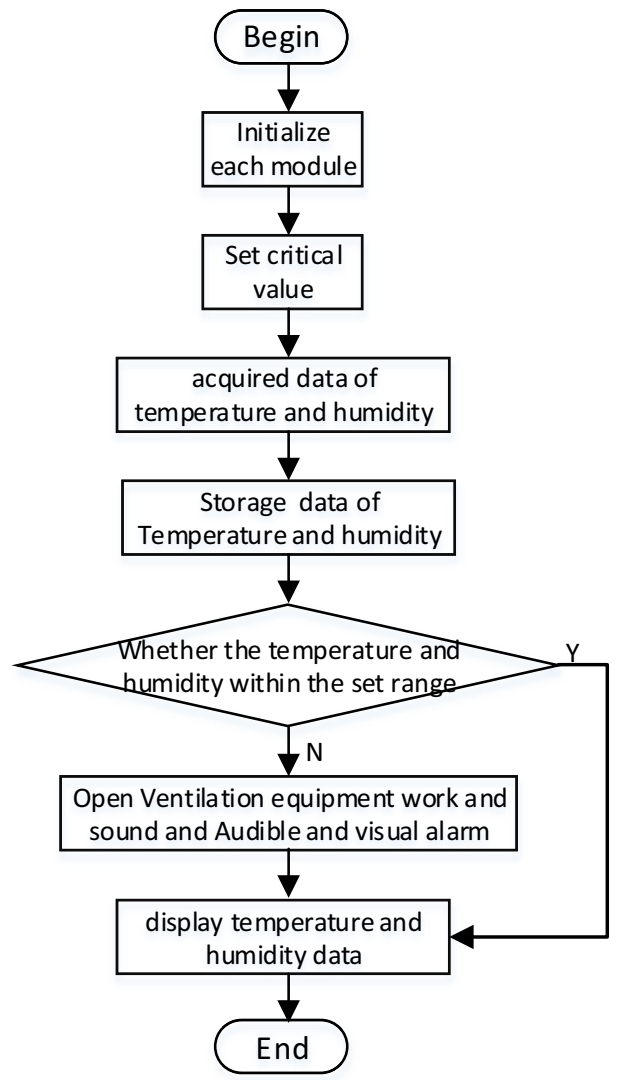

Figure 8. Program flow chart

\subsection{Software design of monitoring center}

Monitoring software is developed using vc ++6.0 , software has friendly interface, convenient operation, the control and monitoring center connect wireless communication circuit to complete information transmission through GSM module TC35i. System is mainly composed of the communication control part ,the parameters of the serial port and the number set parts of SMS service center, collecting data to database, data display and alarm display part, send controlling instruction parts and database maintenance, etc. The monitoring center software is equipped with the temperature display function, the query point to point, temperature anomaly prompt, remote temperature setting, system configuration, and other functions, printing, historical data query, temperature curve shows, etc.

\section{Conclusion}

This paper make full use of the public mobile communication GSM network resources, designed the MSP430 MCU as the core, using temperature and humidity sensor of intelligent detection of granary environmental and food temperature detector, To communicate with wireless monitoring terminal communications composed of TC35i, can control the TC35i send short message to the monitoring center computer, realized the remote monitoring system of granary temperature and humidity, greatly reduces the 
cost. Making grain storage quality guaranteed, this design has wide application prospect and value in the granary storage.

\section{References}

1. Xiao ling XU, Shaobiao Li, Fuqiang Zhang.Design of Granary Temperature and Humidity Control System Based on ZigBee. Journal of Guangdong University of Petrochemical Technology[J].2011(21)

2. LiuYang, Zhihuai Mao, Zhijie Jiang,etc.The granary temperature and humidity remote monitoring system based on wireless transmission.Transactions of the Chinese Society of Agricultural Engineering[J].2012(04)

3. Juncai,Hu hua Hou,HouYao. A Digital Temperature and Humidity Monitoring System for Grain Storage.Journal of Agricultural Mechanization Research[J].2012(09)

4. Wuli Wang, Hua Yang. Design of Granary Temperature and Humidity Measurement and Control System Basedon SHT11Instrument Technique and Sensor [J].2010(09)

5. Chaoze Huang, Qiyi Guo, Zhong-ya Mao.The Design and Implementation of Sending and Receiving SMS Based on MSP430G2231 and TC35i. Electrical Automation[J].2013(01)

6. Fuquan Wang,Pin,Feng Wan,miao Kong. Application of DS18B20 in the temperature collect module of air detection system.Application of Electronic Technique[J].2011(08)

7. Xin Zan; Zhongde Yu. Design of the remote monitoring system of food cold storage based on GSM technology.Journal of Dalian Polytechnic University[J].2014(06) 